\title{
5 Textkomposition im Jalkut Schimoni Zwölfprophetenbuch
}

Die Kommentierung des Jalkut Schimoni zu Hosea bis Maleachi unterscheidet sich von den Kommentierungen des Jalkut Schimoni zur Tora dadurch, dass zu diesen prophetischen Schriften ${ }^{1}$ keine fortlaufenden Midraschim vorliegen, die der Autor für seine Arbeit hätte nutzen können. Er war gezwungen, aus Tausenden von Einzelstücken, entnommen aus den unterschiedlichsten Schriften, eine fortlaufende Auslegung zu erschaffen. Dass er dabei zusätzlich die Mühe auf sich nahm, bestimmte Themen anzusprechen und diese über die zwölf Bücher verteilt in die fortlaufende Kommentierung einzuweben, geschah, um eine inhaltliche Kontinuität zu schaffen, die die Kommentierung der zwölf Einzelschriften von Hosea bis Maleachi zu der Kommentierung des Zwölfprophetenbuches macht. Auch die über die zwölf Schriften sich erstreckende konsistente Auswahl und Positionierung von Quellen zeigt, dass der Jalkut zu Hosea bis Maleachi als ein in sich geschlossener Kommentar konzipiert ist.

\subsection{Textkomposition durch Quellenauswahl}

Die Verwendung der Quelle Seder Olam ist ein Element im Jalkut Schimoni, dass die Kommentierung der zwölf prophetischen Bücher von Hosea bis Maleachi zum Zwölfprophetenbuch zusammenschließt. In sechs von zwölf Büchern, nämlich zu Hosea, Amos, Obadja, Micha, Nahum und Habakuk wird Seder Olam 20 als erste Kommentarquelle verwendet. Zu Hosea, Amos und Micha wird Seder Olam jeweils zu dem ersten Vers des ersten Kapitels als Quelle benutzt. Zu Nahum und Habakuk, die keine Auslegung zu ihren Versen 1,1 bieten, wird trotzdem als erste Quelle Seder Olam 20 angeführt. Obadja bringt Seder Olam 20 als dritte Auslegung zu Ob 1,1. Zu Joel und Jona wird jeweils MidrPs 80,1 verwendet.

Der babylonische Talmud stellt ca. die Hälfte des Quellenmaterials im Jalkut Schimoni zum Zwölfprophetenbuch. Es fällt auf, dass für die Kommentaranfänge von Zephanja und Maleachi jeweils bMeg 15a verwendet wird. Die nähere Betrachtung zeigt, dass die dort verwendeten Quellenstücke genau aneinander anschließen. Löst man also beide Quellenstücke aus dem Kommentar, ergänzen diese sich.

Im Übrigen gilt, dass die Quellen des babylonischen Talmuds stets dem Jerusalemer Talmud vorgezogen werden. Gibt es zu einem Vers mindestens zwei Auslegung

1 Ausgenommen zum Buch Jona. 
und eine stammt aus dem Babylonischen und die andere aus dem Jerusalemer Talmud, werden entweder beide angeführt oder nur die des babylonischen Talmuds.

Um dieses Verfahren zu illustrieren, werden im Folgenden alle Verse aus Jalkut Hosea, zu denen mehr als eine Auslegung aus dem Babylonischen Talmud und/oder auch mindestens eine Auslegung aus dem Jerusalemer Talmud existiert, aufgelistet und gezeigt, welcher der beiden Talmudim jeweils verwendet wird:

\begin{tabular}{|c|c|c|}
\hline Ausgelegter Vers: & Mögliche talmudische Quellen: & Verwendet wird: \\
\hline Hos 2,1 & bPes $87 b$, bJoma 22b, bQid 36a & bJoma 22b \\
\hline $\operatorname{Hos} 2,7$ & $\begin{array}{l}\text { bKet } 65 a \text {, bNazir } 23 a \text {, bHor } 10 b \text {, } \\
\text { jSanh } 2,6\end{array}$ & bBer $32 a^{2}$ \\
\hline Hos 2,12 & bSchab 55a, jSanh 10,1 & bSchab 55a \\
\hline Hos 2,13 & $\begin{array}{l}\text { bSchab 145b, bTaan 29b-30a, } \\
\text { jTaan 4,6 }\end{array}$ & bSchab 145b, bTaan 29b-30a \\
\hline Hos 3,5 & bMeg 17b-18a, jBer 2,4 & bMeg 17b-18a \\
\hline Hos 4,2 & $\begin{array}{l}\text { bGit 52b, bQid 13a, bSchebu } \\
39 a\end{array}$ & bQid 13a \\
\hline Hos 4,4 & $\begin{array}{l}\text { bQid 70b, bSchab 149b, jJeb } \\
8,3 \text {, jQid 4,1 }\end{array}$ & bQid 70b \\
\hline Hos 4,5 & bMQ 17a, bMen 99b & bMQ 17a \\
\hline Hos 4,6 & bJoma 38b, bSota 49a & bJoma 38b \\
\hline Hos 4,11 & bJoma 76b, bGit 68a, jKet 5,11 & bJoma 76b \\
\hline Hos 4,12 & bPes 52b, bSuk 52b & bPes 52b, bSuk 52b \\
\hline Hos 4,14 & bSota 47a-b, jSota 9,9 & bSota 47a-b \\
\hline Hos 5,2 & bSanh 102a, jAZ 1,1 & bSanh 102a \\
\hline Hos 5,7 & bJeb 17a, bQid 70a, jSanh 10,5 & bQid 70a \\
\hline $\operatorname{Hos} 6,2$ & $\begin{array}{l}\text { bRH 31a, bSanh 97a, jBer 5,2, } \\
\text { jTaan 1,1 }\end{array}$ & bRH 31a \\
\hline Hos 6,3 & $\begin{array}{l}\text { bBer 6b, bTaan 4a, jBer 5,1- } \\
\text { 5,2, jTaan 1,1 }\end{array}$ & bBer 6b \\
\hline Hos 6,4 & bBer 59a, bTaan 6b & bTaan 6b \\
\hline Hos 6,8 & bMak 10a, jAZ 5,10 & bMak 10a \\
\hline Hos 7,5 & $\begin{array}{l}\text { bSota 42a, bSanh 103a, bAZ } \\
\text { 18b, jAZ 1,1 }\end{array}$ & bSota $42 a$ \\
\hline Hos 8,12 & bGit 60b, jPea 2,6, jChag 1,8 & bGit 60b, jPea 2,6 \\
\hline Hos 8,14 & jScheq 5,7 (6) & jScheq 5,7 (6) \\
\hline Hos 9,1 & bGit 7a, jMeg 3,2, jSota 5,6 & bGit 7a \\
\hline
\end{tabular}

2 Diese Quelle wird zur Auslegung von Hos 2,7 verwendet, obwohl sie den Vers selbst gar nicht erwähnt, sondern nur Hos 2,10 zitiert. 


\begin{tabular}{lll}
\hline Hos 9,10 & $\begin{array}{l}\text { bBer 56b, bSanh 106a, jSanh } \\
\text { 10,2 }\end{array}$ & bSanh 106a \\
Hos 10,5 & bMeg 25b, bSanh 63b & bMeg 25b \\
Hos 10,12 & bSuk 49b, bBQ 17a, bAZ 5b, & bSuk 49b \\
& bBek 24a & \\
Hos 11,9 & bTaan 5a, bTaan 11b & bTaan 5a \\
Hos 13,4 & bPes 87b, bJeb 17a & bPes 87b \\
Hos 13,15 & bGit 31b & bBB 25a \\
Hos 14,10 & bBB 89b, bHor 10b & bBB 89b \\
\hline
\end{tabular}

Deutlich wird, dass der babylonische Talmud im gesamten Jalkut zum Zwölfprophetenbuch dem Jerusalemer Talmud vorgezogen wird. Der Jerusalemer Talmud wird nur dann als Quelle verwendet, wenn es keine Alternative aus dem babylonischen Talmud gibt. Gibt es diese, wird der Jerusalemer Talmud in wenigen Fällen ergänzend hinzugefügt. Gibt es nur eine passende Stelle im Jerusalemer Talmud, wie beispielsweise $\mathrm{zu}$ Hos 8,14, wird der Jerusalemer Talmud verwendet.

\subsection{Textkomposition durch thematische Fokussierung}

Neben der fortlaufenden Auslegung des Bibeltextes des Zwölfprophetenbuches fällt auf, dass der Jalkut zusätzlich für ihn wichtige Themen in diese Auslegung einfügt. $\mathrm{Zu}$ diesen wiederholt aufgegriffenen Themen gehören u.a.: 1) Der Umgang mit „Fremdvölkern“, 2) die Darstellung der Propheten, 3) die Bedeutung der Schechina und 4) die Frage nach der messianischen Zeit. Diese Themen bilden eine in sich geschlossene thematische Abhandlung von Hosea bis Maleachi, die die Kommentierung der zwölf Bücher zu einem Gesamtkommentar, dem Jalkut zum Zwölfprophetenbuch, werden lässt. Um dies zu illustrieren, werden im Folgenden, zu den als zentral benannten Themen, alle Ausführungen schriftenübergreifend zusammengestellt.

\subsubsection{Die Fremdvölker}

Im biblischen Hebräischen werden die Begriffe נכרים גרים oder, גרים benutzt, um Menschen, die nicht ursprünglich zu „Israel“ gehören, zu bezeichnen. Diese können Israel feindlich oder neutral gegenüberstehen. Die גרים werden in der rabbinischen Interpretation sowohl als „Fremde“, als auch als „Proselyten“ verstanden. Sie werden in der Auslegung zu Hos 2,25 spektakulär positiv gesehen. So heißt es hier, dass Gott von Beginn an den Plan hatte, Fremde zu Israel hinzuzufügen, und dass Israel deswegen nach Babylonien geführt wurde:

3 Diese Quelle wird verwendet, obwohl sie Hos 13,15 nicht zitiert. 
Und ich will sie mir ins Land einsäen. (Hos 2,25) R. Eleazar sagte: Der Heilige, gepriesen sei er, brachte Israel nur nach Babylonien, damit zu ihnen Fremde (גרים) hinzugefügt werden, denn es heißt: Und ich will sie mir ins Land einsäen. (Hos 2,25)

In der Jalkutkommentierung zu Hosea wird hervorgehoben, dass die Proselyten besonders von Gott bedacht werden. Sie, die die Gesetze der Tora freiwillig halten, werden von Gott wie eine besondere Gabe betrachtet. $\mathrm{Zu}$ Hos 14,8 heißt es:

Die in seinem Schatten sitzen kehren zurück. (Hos 14,8) R. Abbahu sagte: Diese sind die Proselyten (הגרים), die kommen und im Schatten des Heiligen, gepriesen sei er, Erbarmen finden. [...] Der Heilige, gepriesen sei er, sagte: Lieb sind mir ihre Namen wie der Wein, der über den Altar gegossen wird.

Die nächste Erwähnung eines besonderen Proselyten findet sich in Jalkut Obadja, der den Propheten selbst als edomitischen Proselyten identifiziert. Er ist ein Proselyt, der als Bote bzw. als Verkünder von Gottes Wort auftritt:

R. Isaak sagte: Der Heilige, gepriesen sei er, sagte: Obadja, der zwischen zwei Frevlern lebte und nicht von ihren Taten lernte, soll kommen und Esau, den Frevler, der zwischen zwei Frommen lebte und nichts von ihren Taten lernte, abstrafen. Efraim Makschaah, ein Schüler von R. Meir, sagte: Obadja war ein edomitischer Proselyt (גר).

Auch die Umkehr von Fremden hin zu Proselyten wird thematisiert und begrüßt. So heißt es in Jalkut Jona zum Ende des ersten Kapitels:

Und sie gelobten, ein jeder und seine Frau, seine Kinder und alles, was er hatte, dem Gott Jonas. Und sie gelobten und erfüllten es. Und über sie heißt es: „Und über die Proselyten der Gerechtigkeit.“4

Zu Zeph 3,9 klärt der Jalkut die Frage, ob die Proselyten Anteil an der kommenden Welt haben werden:

Dann verwandle ich den Völkern (עמים) ihre Lippen. (Zeph 3,9) Dies lehrt, dass er alle Völker selbst ernannte, Proselyten für die kommende Welt zu werden. R. Abaje sagte: Vielleicht werden sie sich nur vom Götzendienst abwenden? Er sagte zu ihm: Es steht geschrieben: Dass sie ihm mit einem Nacken dienen. (Zeph 3,9) In früheren Zeiten stand geschrieben: Sodann, lasst uns hin abfahren und ihre Sprache verwirren. (Gen 11,7) Und es steht geschrieben: JHWH vernichte sie und teile ihre Sprache. (Ps 55,10) Aber in der kommenden Welt: Dann wandle ich den Völkern ihre Lippen. (Zeph 3,9)

In Jalkut Mal 3,5 wird schließlich die rechtliche Gleichstellung der Proselyten erklärt:

Ich werde an euch herantreten, zum Gericht, und werde ein schneller Zeuge sein. (Mal 3,5)

R. Jochanan weinte, wenn er an den Schriftvers kam. Er sagte: [Das gleicht] einem Sklaven, den

4 Die 18. Bitte des Achtzehnbittengebets. 
sein Herr vor Gericht bringt und gegen ihn Zeugnis ablegt. Gibt es ein Mittel für ihn? R. Jochanan sagte: Wehe uns, dass [die Schrift] uns Leichtes wie Schweres anrechnet. R. Levi sagte: Jeder, der das Recht eines Proselyten (גר) beugt, ist so, als würde er das Recht des Höchsten beugen, denn es heißt: Die das Recht des Fremden beugen. (Mal 3,5) „Die mich beugen“ steht geschrieben. R. Chanina bar Papa sagte: Jeder, der etwas [Falsches] tut und es bereut, dem vergibt man sofort, denn es heißt: Und fürchte dich nicht vor mir. (Mal 3,5) Aber, wenn sie sich vor mir fürchten, vergibt man ihnen.

In deutlichem Kontrast zu der Gruppe der Proselyten sieht der Jalkut die Gruppe der Fremden, die als „Völker der Welt“ bezeichnet wird. Sie stehen konsequent negativ konnotiert als Gegengruppe zu Israel. Der Autor des Jalkut stellt diese Gruppe gleichbleibend durch den Kommentar zum Zwölfprophetenbuch als Gruppe der Widersacher dar. Während die „Fremden“ eine undefinierte Gruppe sind, die nicht einer bestimmten Identität zugeordnet werden und auch als heterogene Gruppe aus verschiedenen Herkünften zusammengesetzt sein können, beschreibt die Gruppe der „Völker“ in der Regel eine spezifische Gruppe. In der Folge wird diese Gruppe dann häufig als Babylonier oder eine andere götzendienende Gruppe identifiziert.

Der Jalkut thematisiert zu Hos 9,1, dass Israel nicht die Kultur der andern Völker übernehmen solle:

Freue dich nicht, Israel, rühme dich nicht wie die Völker (כעמים). (Hos 9,1) Sie sandten [eine Frage] an Mar Uqba: Woher haben wir [den Beleg], dass Musik verboten ist? Er linierte [einen Brief] und schrieb: Freue dich nicht Israel, rühme dich nicht wie die Völker. (Hos 9,1) Er hätte ihnen von hier [eine Antwort] schicken sollen: Nicht mehr wird bei Gesang Wein getrunken, bitter ist der Rauschtrank für seinen Trinker. (Jes 24,9) Wenn von hier, könnte ich meinen, diese Worte [gelten] nur bei Musik mit einem Instrument, mit dem Mund aber sei es erlaubt. [Dass dem nicht so ist,] das lehrt er uns.

Die Auslegung zu Joel 3,4, gezeigt, dass es sich bei den Völkern immer auch um Götzendiener handelt:

Und wofür ich würdig wäre, gepriesen zu werden, [damit] verärgern mich die Völker (האומות): Dieser sagt: Ich diene den Sternen. Und jener sagt: Der Sonne und dem Mond diene ich. Deshalb erschüttere ich sie, denn sie haben mich durch sie verärgert. Deshalb leiden auch sie mit ihnen, denn es heißt: Die Sonne wird sich in Finsternis verwandeln und der Mond in Blut. (Joel 3,4)

Von Joel fortsetzend, finden sich weitere Beschreibungen der Eigenschaften der Völker in Jalkut Micha. Zu Mi 6,3 werden die Völker als deutlich feindselig beschrieben:

R. Samuel bar Rav Nachman sagte: An drei Stellen kam der Heilige, gepriesen sei er, um mit dem Volk (עם) Israel zu argumentieren, und die Völker (עומות) der Welt freuten sich und sagten: Wie kann dieses Volk (עם) mit seinem Schöpfer argumentieren? Jetzt wird er sie vernichten.

Weiter heißt es in der Auslegung zu Mi 6,7: 
Bileam, der Frevler, war der Verteidiger der Völker (אומות) der Welt und über die Völker der Welt sagte er: Hat JHWH Gefallen an Tausenden Widdern? (Mi 6,6-7)

Im Jalkut Schimoni werden die Völker der Welt zudem als Strafe Gottes gesehen bzw. deren Handeln als Strafandrohung Gottes dargestellt, und es wird deutlich, dass Gott mehrfach als Beschützer der Israeliten vor den Völkern der Welt auftritt. So heißt es in Jalkut Schimoni zu Mi 7,16:

Gott sagte: Wenn ihr meinen Willen tut, werde ich sie, genauso wie ich die Schlange beschämt und verflucht habe [beschämen und verfluchen], denn es heißt: Verflucht seist du vor allem Vieh. (Gen 3,14) In der Zukunft beschämen und verfluchen, denn es heißt: Völker werden sehen und sie werden sich schämen. $(M i$ 7,16) Und wenn sie nicht meinen Willen tun: Wie die Schlange Adam und Eva bekämpfte und sie aus der Welt vertrieb, so werde ich euch Völker (אומות) der Welt bekämpfen, denn sie gleichen Schlangen, denn es heißt: Ihre Stimme ergeht wie die einer Schlange. $(J e r 46,22)$ Und ebenso heißt es: Wenn die Schlage sticht, weil keine Beschwörung da war. (Koh 10,11)

Die Völker waren zudem unwürdig, die Tora zu empfangen, wie zu Hab 3,6 erklärt wird:

Der Heilige, gepriesen sei er, maß alle Völker (האומות) und befand kein Volk für würdig, die Tora zu empfangen, außer Israel.

Den Völkern der Welt bleibt zudem die mündliche Tora verwehrt, wie in Jalkut Schimoni zu Hosea 8,12 erklärt wird:

Ich werde ihm das meiste meiner Lehre aufschreiben. (Hos 8,12) Diese sind die Zurechtweisungen, sogar so: Wie ein Fremder sind sie geachtet. (Hos 8,12) R. Abin sagte: Wenn ich dir das meiste meiner Lehre aufgeschrieben hätte, wären sie nicht wie ein Fremder geachtet gewesen. Was [ist der Unterschied] zwischen dir und den Völkern der Welt? Diese ziehen ihre Bücher hervor, und jene ziehen ihre Bücher hervor. R. Chaggai, Sohn von R. Simlai [sagte]: Es steht geschrieben: Denn wegen des Mündlichen dieser Worte habe ich mit dir einen Bund geschlossen. (Ex 34,27) Dies heißt: Jene, die mündlich sind, sind die beliebten. [...] R. Jehuda bar Schalom sagte: Mose wollte, dass die Mischna aufgeschrieben werde, und der Heilige, gepriesen sei er, sah voraus, dass die Völker (אומות) der Welt die Tora übersetzen und sie in Griechisch ('I $\omega v i$ í $\alpha$ ) lesen und sagen würden, dass auch sie Israel seien. [Dann] sagt der Heilige, gepriesen sei er, zu den Völkern (אומות): Wieso sagt ihr, dass ihr mein Kind seid?! [Davon] weiß ich nichts. Vielmehr, wer meine Geheimnisse in seiner Hand hat, ist mein Kind. Und was sind die Geheimnisse? Das ist die Mischna. Denn wenn die Mischna in der Hand der Völker wäre, [dann] wäre [es] so: Wie ein Fremder sind sie geachtet. (Hos 8,12)

Ammoniter und Moabiter, als Teil der Völker der Welt, werden zu Zeph 2,9 als besondere Feinde beschrieben:

Du findest, als die Sünden es verursachten, dass die Völker (גוים) in Jerusalem einfielen, fielen auch die Ammoniter und Moabiter mit ihnen ein. Und sie fielen in den Tempel ein, nahmen die Cherubim und ließen sie durch die Straßen Jerusalems ziehen. Und sie sagten: Hat nicht Israel 
gesagt: Wir treiben keinen Götzendienst?! Seht was sie tun. Dies ist es, was geschrieben steht: Weil Moab spricht: Siehe, das Haus Juda ist so wie alle Völker (גוים). (Ez 25,8) In dieser Stunde sagte der Heilige, gepriesen sei er: Dass Moab wie Sodom sein werde. (Zeph 2,9)

Eine Ausnahme unter den Völkern der Welt bietet die Gruppe der Gerechten, die aber nicht zu den Proselyten zählen. Von dieser Gruppe heißt es, dass sie für das Fortbestehen der Völker der Welt verantwortlich ist. Dies klärt die Frage, warum die Völker der Welt nicht einfach von Gott zerstört werden. Da unter ihnen Gerechte leben, sorgen sie für deren Fortbestand. So heißt es in Jalkut zu Sach 11,12:

Und ich sprach zu euch: Wenn es in euren Augen gut ist, gebt meinen Lohn, und wenn nicht, lasst es. Und ich wog dreißig Silberlinge ab. (Sach 11,12) Rav Jehuda sagte: Dies sind die dreiBig Gerechten der Völker (אומות) der Welt, um derentwillen die Völker (אומות) der Welt bestehen bleiben.

Eine letzte Anmerkung zu den Völkern der Welt findet sich in Jalkut Mal 2,16. Während vorausgehend Israel als auserwählt herausgehoben wurde, wird hier betont, dass durch das Auserwähltsein Israel Rechtsvorschriften übertragen wurden:

Israel gab ich die Scheidung, nicht den Völkern (אומות).

Die Unterscheidung zwischen den Völkern der Welt und den Proselyten bleibt von Hosea bis Maleachi hinweg im Jalkut gleich und ist damit ein Beispiel für ein schriftenübergreifendes Thema innerhalb des Jalkut Schimoni Zwölfprophetenbuch.

\subsubsection{Die Propheten}

$\mathrm{Zu}$ Beginn von Jalkut Hosea wird Hosea zusammen mit Amos, Micha und Jesaja historisch eingeordnet. Dabei findet keine Bewertung statt; es geht ausschließlich um die Frage, welcher Prophet zu welcher Zeit lebte. Die lokale Situation der Propheten, ob sie im Nordreich oder in Babylonien im Exil leben, spielt ebenfalls keine Rolle.

Wie Propheten und ihr Handeln im Jalkut Schimoni bewertet werden, zeigt sich schon im ersten Kapitel von Jalkut Hosea. Hier werden die Propheten als die Gegenspieler von Königen gesehen, die als von Gott direkt gesandt gelten. In der Auslegung zu Hos 1,1 heißt es:

R. Jochanan sagte: Wehe der Herrschaft, die ihre Besitzer begräbt, denn du hast keinen Propheten, der nicht vier Könige besiegt hätte, denn es heißt: In den Tagen Usias, Jotams, Ahas, Hiskias. (Hos 1,1) Und ebenso heißt es: Vision des Jesaja. (Jes 1,1) R. Jochanan sagte: Warum war Jerobeam, Sohn des Joasch, würdig, zu den Königen des Hauses Davids gezählt zu werden? Weil er das schlechte Reden über Amos nicht übernahm. Woher [ist zu belegen], dass er [dazu] gezählt wurde? Weil geschrieben steht: Wort JHWHs zu Hosea, Sohn des Beeri, in den Tagen Usias, Jotams, Ahas, Hiskias, Könige von Juda, und in den Tagen Jerobeams, Sohn Joaschs, König von Israel. (Hos 1,1) Und woher ist zu belegen, dass er es nicht annahm? Weil geschrieben steht: Und Amazia, der Priester von Bet-El, sandte zu Jerobeam, dem König von Israel, um zu sagen: Amos hat 
sich gegen dich verschworen, inmitten des Hauses Israel. Das Land kann all seine Worte nicht ertragen. Denn so spricht Amos: Jerobeam wird durch das Schwert sterben, und Israel wird aus seinem Land gefangen weggeführt werden. (Am 7,10-11) Er sagte zu ihm: Gott bewahre, dass der Rechtschaffene dies gesagt hat! Und wenn er es gesagt hat, was sollte ich ihm tun? Die Schechina hat es ihm gesagt.

Während Könige und auch Priester fehlbar sein können, sind die Propheten unfehlbar, wenn sie als Übermittler des Gotteswortes fungieren, da sie in direktem Kontakt mit Gott stehen. Sind sie fehlbar, stammt dies aus ihrem Handeln als Mensch. Diese von Propheten begangenen Fehler werden allerdings immer korrigiert und zeugen wie bei Jona - als Vorbild der Umkehr für alle anderen Menschen. Propheten wird es daher auch in der kommenden Welt geben, wie aus Jalkut Hos 2,17 hervorgeht:

Und ich gebe ihr Weinberge von dort. (Hos 2,17) [Der Vers] spricht von Sodom und Gomorra, denn es heißt: Und er zerstörte diese Städte. (Gen 19,25) Und es steht geschrieben: An den harten Stein legt er seine Hand, kehrt ihn um vom Fuße der Berge. (Ijob 29,9) In der kommenden Welt, wird der Heilige, gepriesen sei er, Israel heilen und [auch] sie heilen, denn es heißt: Und ich gebe ihr Weinberge von dort. (Hos 2,17) Das sind die Propheten.

Auch in Jalkut Hos 6,1 bringt der Prophet Israel die Bedeutung der Umkehr in Erinnerung:

Denn er hat uns zerrissen, und er wird uns heilen. (Hos 6,1) [Das gleicht] jemandem, dem ein Geschwür aufgewachsen ist, das der Arzt öffnet. Nachdem er sah, dass sein Geschwür nicht heilt, ging er zu anderen Ärzten, aber er fand keinen [besseren] Arzt. Sie sagten zu ihm: Geh zu dem Arzt, der dir das Geschwür hat aufwachsen lassen, er wird dich heilen. Ebenso sagte der Prophet: Immer, wenn ihr euch von dem Heiligen, gepriesen sei er, entfernt, sind Tröstungen fern von euch. Vollzieht vielmehr eine Umkehr, und der Heilige, gepriesen sei er, wird euch erlösen. Er, der geschlagen hat, ist es, der heilt. Ich verwunde und ich heile. (Dtn 32,39) Ich bin es, der geschlagen hat, denn es heißt: Aus der Höhe schickt er Feuer. (Thr 1,13) Und ich bin es, der heilt: Ich, ich bin es, der euch tröstet. (Jes 51,12)

Dass es auch unter den Propheten hierarchische Unterschiede gibt, zeigt sich in der Unterscheidung zwischen Mose und allen anderen Propheten. Mose war der bedeutendste aller Propheten, denn nur er stand in diesem direkten Kontakt zu Gott, während alle anderen Propheten nur mittelbaren Kontakt zu Gott durch Visionen oder Auditionen hatten. In Jalkut Hosea wird dies durch das Bild des Spiegels symbolisiert. In der Auslegung zu Hos 12,11 heißt es:

Und durch die Propheten lasse ich in Gleichnissen reden. (Hos 12,11) R. Jehuda, Sohn von R. Ilai und die Rabbinen. R. Jehuda sagt: Alle Propheten sahen in sieben Spiegel (speculum), wie du gesagt hast: Und es war anzusehen wie das Gesicht, das er gesehen hatte. (Ez 43,3) Und Mose sah in einem Spiegel (speculum), denn es heißt: In einer Vision und nicht in Rätseln. (Num 12,8) Und die Rabbinen sagen: Alle Propheten sahen in einen beschlagenen Spiegel (speculum) und Mose sah in einen klaren Spiegel. In Bezug auf die Propheten steht geschrieben: Und ich, eine Vision habe ich vermehrt, und durch die Propheten lasse ich in Gleichnissen reden. (Hos 12,11) Und in Bezug auf Mose steht geschrieben: Das Bildnis JHWHs erblickte er. (Num 12,8) R. Pinchas im 
Namen R. Hoschaja: [Das gleicht] einem König, der sich seinem Haussohn [nur] in seinem Bild zeigte. In dieser Welt zeigt sich die Schechina [nur] den Verwaisten, aber in der kommenden Welt wird sich die Ehre JHWHs zeigen und alles Fleisch wird sie gemeinsam sehen. (Jes 40,5) [...] Da floh Jakob [in die] Gefilde Arams. (Hos 12,13) Der Prophet sagte zu Israel: Euer Beispiel gleicht Jakob: Wie Jakob, bevor er eine Frau geheiratet hatte, verknechtet worden war, und nachdem er eine Frau geheiratet hatte, verknechtet worden war, [so] auch ihr: Bevor ein Erlöser geboren ist, seid ihr verknechtet, und nachdem ein Erlöser geboren worden ist, seid ihr verknechtet. Und durch einen Propheten führte JHWH Israel aus Ägypten herauf. (Hos 12,14)

Die Propheten sind Autoritätspersonen, denen Israel sein Vertrauen schenkt. Sie weissagen und halten ihr Wort, ihre Vorhersagen treffen ein. In Jalkut Joel 1,4 sagt der Prophet den Regen voraus:

Was die Raupe ließ, fraß die Heuschrecke. (Joel 1,4) R. Jochanan sagte: In den Tagen Joels wurde dieser Vers erfüllt. In diesem Jahr verging [der] Adar, [aber] kein Regen fiel. Der erste Regen fiel für sie am ersten Nisan. Der Prophet sagte zu [Israel]: Geh und säe! Es sagte zu ihm: Wer Weizen und Gerste hat, soll sie essen und überleben oder aussäen und sterben?! Er sagte zu ihm: Dennoch, geh und säe! Sie gingen und säten. Ihnen wiederfuhr ein Wunder und sie entdeckten [Getreidekörner], die in den Ameisenhügeln und in Wänden [versteckt] waren. Sie gingen und säten am dritten und vierten [Nisan], und ein zweiter Regen kam für sie am fünften Nisan herab, und am sechszehnten brachten sie die Erstlingsgarbe dar. Du findest, dass das Getreide, das [normalerweise] in sechs Monaten wächst, in elf Tagen wuchs. Du findest, dass die Erstlingsgarbe, die man [normalerweise] von Getreide darbringt, das sechs Monate [wächst], von Getreide darbrachte, das elf Tage [gewachsen war]. Und über diese Generation heißt es: Die mit Tränen säen, ernten mit Jubel. (Ps 126,5)

Dem Propheten werden Geheimnisse von Gott geoffenbart. In Jalkut Am 3,7 wird dazu hervorgehoben, dass ein Prophet eine Botschaft immer direkt von Gott empfängt und in seinem Auftrag handelt, um diese Botschaft zu verbreiten:

Denn JHWH, der Gott, wird nichts tun, [er hätte denn sein Geheimnis offenbart]. (Am 3,7) 1) Das ist Josef: Und es sprach Josef zum Pharao. (Gen 41,25) Eine andere Auslegung: 2) Das ist Daniel: Damals wurde Daniel in einem Traumgesicht das Geheimnis offenbart. (Dan 2,19) Eine andere Auslegung: 3)Das ist Elischa: Aber JHWH hat mir gezeigt, dass er sterben wird. (II Reg 8,10) Eine andere Auslegung: 4) Dies sind die Weisen[, denn wir haben gelernt]: In den vier-Abschnitten ist die Sache ausgeführt. Eine andere Auslegung: 5) Das ist Elija: Und Jehu, den Sohn des Nemschi, sollst du zum König salben. (I Reg 19,16) Eine andere Auslegung: 6) Das ist Ahia aus Schilo: Siehe, die Frau Jerobeams. (I Reg 14,5) Eine andere Auslegung: 7) Das ist Samuel: Und JHWH hatte Samuels Ohren offenbart [...], morgen zu dieser Stunde werde ich einen Mann zu dir senden (I Sam 9,15-16) Als erstes war das Geheimnis JHWHs denen, die ihn fürchten, denn es heißt: Das Geheimnis JHWHs, denen, die ihn fürchten. (Ps 25,14) Danach den Standhaften, denn es heißt: Und sein Geheimnis ist mit den Standhaften. (Prov 3,32) Danach den Propheten: Denn JHWH, der Gott, wird nichts tun[, er hätte denn sein Geheimnis offenbart]. (Am 3,7) Der Heilige, gepriesen sei er, sagte: Abraham ist ein Prophet, denn es heißt: Gib dem Mann die Frau zurück, denn er ist ein Prophet. $($ Gen 20,7) Und ich sollte [es] ihm nicht offenbaren? Und JHWH sprach: Wie kann ich vor Abraham verbergen? (Gen 18,17)

In Jalkut Schimoni zu Am 8,12 heißt es: 
Das Wort JHWHs. (Am 8,12) Das ist die Prophetie.

Jona hingegen ist ein besonderer Prophet, da er sich weigerte, das an ihn ergangene Wort Gottes auszuführen. Aber der Jalkut betont zu Jona Kapitel 1, dass er, wie die übrigen Propheten auch, anbot, sein Leben für Israel zu geben:

Jona ging nur, um sich im Meer zu vernichten, denn es heißt: Nehmt mich und werft mich ins Meer. (Jon 1,12) Und so findest du [es] bei den Vätern und bei den Propheten, die ihr Leben für Israel gaben.

In einer Liste mit sechs Einträgen wird in Jalkut Micha und damit in der Mitte des Zwölfprophetenbuches hervorgehoben, dass alle Propheten, wenn sie eine Drohung auszusprechen hatten, eine Tröstung hinzufügten. In Jalkut Mi 7,18 heißt es:

1) Weil Mose Israel harte Worte sagte: Auszehrung durch Hunger und Hinraffen durch Fieber. (Dtn 32,24) Von draußen wird das Schwert aufreiben. (Dtn 32,25) Am Horeb machtet ihr zornig. (Dtn 9,8) Und bitter wart ihr, (Dtn 9,24) kam er zurück und sagte ihnen Worte des Trostes: Und dies ist der Segen. (Dtn 33,1) 2) Und ebenso findest du es bei Joel, dass er ihnen zuerst harte Worte sagte: Ist dies geschehen in euren Tagen? [...] Über dies erzählt euren Kindern. (Joel 1,2-3) Was die Raupe lie $\beta$, fraß die Heuschrecke. (Joel 1,4) Er kam zurück und sagte ihnen Worte des Trostes: Und ich werde euch die Jahre erstatten. (Joel 2,25) 3) Und von ihm lernten alle Propheten. Und ebenso sagte Hosea zuerst seine Worte: Gib ihnen JHWH. Was willst du ihnen geben? Gib ihnen einen fehlgebärenden Schoß. (Hos 9,14) Er kam zurück und sagte ihnen Worte des Trostes: Seine Zweige gehen hervor. (Hos 14,7) Die in seinem Schatten sitzen kehren zurück. (Hos 14,8) Ich will ihre Abtrünnigkeit heilen. (Hos 14,5) Ich will wie der Tau für Israel sein. (Hos 14,6) 4) Und ebenso sagte Amos: Hört diese Worte, Kühe Baschans, im Gebirge Samarias, die ihr die Armen bedrückt. (Am 4,1) Er kam zurück und sagte ihnen Worte des Trostes. 5) Und ebenso sagte Micha ihnen: Wer ist ein Gott wie du? Der Sünde aufhebt und Übertretung übergeht. Dem Überrest [seines Eigentums]. (Mi 7,18) Wiederholt wird er sich unser Erbarmen. (Mi 7,19) Du wirst Jakob Treue erweisen. (Mi 7,20) 6) Und ebenso sagt Jeremia: Und ich mache der Stimme der Lust und der Stimme der Freunde ein Ende in den Städten Judas und den Straßen Jerusalems. (Jer 7,34) Und er kam zurück und sagte ihnen Worte des Trostes: Und es freut sich die Jungfrau. (Jer 31,13) 7) Man könnte meinen, dass, nachdem er ihnen Worte des Trostes gesagt hatte, kehrten sie um und sagten ihnen Worte der Mahnung. Die Bibel lehrt: Also wird Babel versinken und sich nicht sammeln. (Jer 51,64) Siehe, nachdem er ihnen Worte des Trostes gesagt hatte, kam er nicht zurück und sagte ihnen Worte der Mahnung.

Mit der Auslegung zu Zeph 1,1 erklärt der Jalkut, warum Namen und Herkunft der Propheten unterschiedlich genau angegeben werden:

Das Wort JHWHs, das an Zephanja, den Sohn Kuschis, Sohn von Gedalia erging. (Zeph 1,1) Ula sagte: Bei jedem, bei dem Namen und der Name seines Vaters bei Prophezeiungen ausdrücklich genannt sind, ist sicher, dass er Prophet, Sohn eines Propheten ist. Sein Name, aber nicht der Name seines Vaters, ist sicher, dass er Prophet, nicht aber der Sohn eines Propheten ist. Bei jedem, dessen Name und der Name seiner Stadt ausdrücklich genannt sind, ist sicher, dass er ein Prophet aus dieser Stadt ist. Sein Name, aber nicht der Name seiner Stadt, ist sicher, dass er aus Jerusalem ist. In einer Baraita wird gelehrt: Bei jedem, dessen Werke und die Werke seiner 
Vorväter nicht ausdrücklich genannt sind, und die Schrift dir einen von ihnen lobend hervorhebt, wie: Das Wort JHWHs, das an Zephanja erging, (Zeph 1,1) ist sicher, dass er ein Gerechter, Sohn eines Gerechten ist. Bei jedem, dessen Werke und die Werke seiner Vorväter nicht ausdrücklich genannt sind, und die Schrift dir einen von ihnen beschämend hervorhebt, wie: Und im siebten Monat kam Ismael, der Sohn Netanias, (Jer 41,1) ist sicher, dass er ein Frevler, Sohn eines Frevlers ist. Steht geschrieben in [Jalkut] Könige in \$252 [bMeg 15a, Jalkut Könige]. Zephanja war einer von drei Propheten, der in seiner Generation prophezeite: Jeremia, Zephanja und Hulda, die Prophetin. Jeremia prophezeite auf dem Marktplatz, Zephanja prophezeite in den Versammlungshäusern und in den Lehrhäusern, und Hulda bei den Frauen. Und dennoch: Und sie hörten nicht und sie neigten nicht ihr Ohr. (Jer 7,24)

In Jalkut Hag 1,13 wird die Doppelbedeutung „Bote“ und „Engel“ des Wortes מלאך vorgeführt, da Propheten als Boten Gottes gelten:

Da sagte Haggai[, der Bote (מלאך) JHWHs] mit der Botschaft (מלאכות) JHWHs. (Hag 1,13) Du findest [hier], dass die Propheten „Engel“ (מלאכים) genannt werden. Dementsprechend: Lobt JHWH, seine Engel (מלאכיו). (Ps 103,20) Dementsprechend: Und er sandte einen Engel (מלאים). (Num 20,16) Dementsprechend: Und sie verspotteten die Engel (מלאכי) Gottes. (II Chr 36,16) Dementsprechend: Und es kam der Engel (מלאך) JHWHs herauf von Gilgal nach Bochim. (Jdc 2,1)

In Jalkut Mal 1,1 wird darüber diskutiert, ob der letzte Prophet des Zwölfprophetenbuches, Maleachi, in die Zeit des Darius zu datieren ist:

Vortrag des Wortes JHWHs an Israel durch Maleachi (מלאכי). (Mal 1,1) Rav sagte: Maleachi ist Mordechai. Und warum wird er Maleachi genannt? Weil er Vizekönig (משנה למלך) war. Man wandte ein: Baruch, der Sohn Nerijas und Seraja, der Sohn Machseja und Daniel, der ehrenwerte Mann, und Mordechai, Bilaschan und Haggai, Sacharja und Maleachi, sie alle prophezeiten im zweiten Regierungsjahr des Darius. Meint man wirklich, [es ist] Mordechai und meint man wirklich, [es ist] Maleachi? Eine Widerlegung: Es wird gelehrt: R. Jehoschua ben Qarcha sagt: Maleachi, das ist Esra. Und die Weisen sagen: Sein Name war [tatsächlich] Maleachi. Rav Nachman bar Jitzchaq sagte: Es ist logisch nach dem, der sagte, Maleachi ist Esra, denn über die Prophetie Maleachis steht geschrieben: Treulos ist Juda und Abscheuliches ist in Israel geschehen. [Er liebt und heiratet die Tochter eines fremden Gottes]. (Mal 2,11) Und wer sonderte die fremden Frauen von Israel aus? Esra, denn es steht geschrieben: Da antwortete Schechanja, Sohn Jechiels [...], und er sagte zu Esra: Wir sind gegen JHWH treulos geworden und besitzen fremde Frauen. (Esra 10,2)

Die letzte Anmerkung über Propheten in Jalkut Maleachi 3,16 beschließt das Thema mit der nüchternen Erkenntnis, dass es keine Propheten mehr gibt. Allerdings werden in der Zukunft Elija und der Messias kommen, die beide ,alles aufschreiben werden“, wie die Propheten es zu tun pflegten:

R. Cohen sagte im Namen R. Levis: In der Vergangenheit, wenn ein Mensch ein gutes Werk getan hatte, schrieben die Propheten es auf: Und als Ruben es hörte, rettete er ihn aus ihrer Hand. (Gen 37,21) Und er reichte ihr geröstetes Getreide. (Rut 2,14) Jetzt gibt es keine Propheten mehr. Wer schreibt es auf? Elija und der König Messias und der Heilige, gepriesen sei er, siegelte es auf ihre Hände, denn es heißt: Einst besprachen sich die, die JHWH fürchten, miteinander. (Mal 3,16) 
Deutlich wird, dass hier klar dagegen argumentiert wird, dass Gott neue Propheten berufen werde. Die Propheten gehören zu einer vergangenen Zeit. Im Gegensatz dazu entwickelt der Jalkut ein dynamisches Bild für die Schechina.

\subsubsection{Die Schechina}

Zur Zeit des zweiten Tempels ist die Schechina klar im Allerheiligsten des Tempels zu lokalisieren, zu dem nur der Hohe Priester Zugang hat. ${ }^{5}$ In Jalkut Habakuk heißt es in der Auslegung zu Hab 2,20:

JHWH ist in der Halle seines Heiligtums. (Hab 2,20) R. Samuel bar Nachmani sagte: Bevor der Tempel zerstört wurde, war die Schechina in der Tempelhalle.

Im vierten Kapitel zu Micha erklärt der Jalkut aber, die Schechina sei nicht an den Tempel gebunden. Dort heißt es zu Mi 4,10:

Denn du musst aus der Stadt ausziehen und auf dem Feld (שדה) leben. (Mi 4,10) Obwohl der Tempel zerstört wurde, wich die Schechina nicht von dort. „Ich wohne“ steht geschrieben. Und „Feld“ (שדה) ist nichts anderes als „Zion“, denn es heißt: Zion soll als Feld (שדה) gepflügt werden. (Mi 3,12) Und so lehren unsere Lehrer: ${ }^{6}$ WENN EIN VERSAMMLUNGSHAUS ZERSTÖRT IST, KLAGT MAN NICHT DARIN UND KNÜPFT KEINE SEILE DARIN.

Dass die Schechina nach der Zerstörung des Tempels weiterhin als Überbringerin einer Prophetie wirksam war, wird direkt zu Beginn der Kommentierung zum Zwölfprophetenbuch geklärt. Dort heißt es zu Hos 1,1, die Schechina habe zu Amos gesprochen:

Denn so spricht Amos: Jerobeam wird durch das Schwert sterben, und Israel wird aus seinem Land gefangen weggeführt werden. (Am 7,10-11) Er sagte zu ihm: Gott bewahre, dass der Rechtschaffene dies gesagt hat! Und wenn er es gesagt hat, was sollte ich ihm tun? Die Schechina hat es ihm gesagt.

Sie lässt sich aber auch auf Menschen nieder, die keine Propheten sind, wie aus der folgenden Negativliste aus Jalkut zu Hos 7,5 von Personen, die die Schechina nicht empfangen zu schließen ist:

Rav Chisda sagte, R. Jirmeja bar Abba habe gesagt: Vier Gruppen [von Menschen] empfangen das Angesicht der Schechina nicht: Die Gruppe der Spötter, der Schmeichler, der Lügner und derjenigen, die mit böser Zunge reden. „Die Gruppe der Spötter“, denn es steht geschrieben: $E r$ entzieht seine Hand den Spottenden. (Hos 7,5) „Die Gruppe der Schmeichler“, denn es steht geschrieben: Vor sein Angesicht kommt kein Schmeichler. (Ijob 13,16) „Die Gruppe der Lügner“,

5 Alan Unterman, „Shekhinah“, Encyclopaedia Judaica, hg. Michael Berenbaum, Fred Skolnik, 2. Aufl., Bd. 18, (Detroit: Macmillan Reference USA, 2007), 440-444.

6 Mischna Meg 3,3. 
denn es heißt: Wer Lügen redet, soll vor meinen Augen nicht bestehen. (Ps 101,7) „Die Gruppe derjenigen, die mit böser Zunge reden“, denn es steht geschrieben: Denn du bist nicht ein Gott, der an Frevel Wohlgefallen hat, Böses darf nicht bei dir weilen. (Ps 5,5)

Nach Jalkut zu Mi 3,12 halten bestechliche Richter die Schechina ganz von Israel fern:

Daher wird euretwegen Zion zum Feld gepflügt. (Mi 3,12) Und der Heilige, gepriesen sei er, lässt seine Schechina nicht eher auf Israel ruhen, bis die Richter aus Israel aufgehört haben[, bestechlich zu sein], denn es heißt: Und ich wende meine Hand gegen dich und ich werde dein unedles Metall läutern. (Jes 1,25)

Dass sich die Schechina weitestgehend zurückgezogen hat, wird in Jalkut Hos 12,11 betont:

R. Pinchas im Namen R. Hoschaja: [Das gleicht] einem König, der sich seinem Haussohn [nur] in seinem Bild zeigte. In dieser Welt zeigt sich die Schechina [nur] den Verwaisten, aber in der kommenden Welt wird sich die Ehre JHWHs zeigen und alles Fleisch wird sie gemeinsam sehen. (Jes 40,5)

Sie wird in der kommenden Zeit aber wieder auf den Gerechten ruhen, wie der Jalkut zu Hos 14,6 hervorhebt:

So wie Tau für kein Geschöpf schädlich ist, so werden einst die Gerechten sich am Glanz der Schechina sättigen, und es wird ihnen nicht schaden, denn es heißt: Sättigungen in Freuden ist [vor] deinem Angesicht. (Ps 16,11)

Dies ist deswegen so, weil die Gerechten durch das Halten der Gebote, die Schechina anziehen. In Jalkut Hab 3,4 heißt es:

Und ein Glanz war wie Licht. (Hab 3,4) Rava sagt: Womit sind die Gerechten vor der Schechina zu vergleichen? Einer Lampe vor der Fackel. Und Rava sagte: Eine Fackel für die Havdala ${ }^{7}$ [zu benutzen] ist die vorzüglichste [Weise] das Gebot [auszuüben].

Ebenso ist die Schechina anwesend, wenn man sich mit der Tora beschäftigt, wie der Jalkut zu Am 9,6 betont:

Und sein Gewölbe hat er über der Erde gegründet. $(A m$ 9,6) R. Chalafta aus Kefar Chanania sagt: Bei zehn, die zusammensitzen und sich mit der Tora beschäftigen, verweilt die Schechina, denn es heißt: Gott steht in der Gemeinde Gottes. (Ps 82,1) Woher [ist zu belegen], sogar bei fünfen? Weil es heißt: Mitten unter Göttern richtet ${ }^{8}$ er. (Ps 82,1) Woher [ist zu belegen], sogar bei dreien? Weil es heißt: Und sein Gewölbe hat er über der Erde gegründet. (Am 9,6) Woher [ist zu belegen], sogar bei zweien? Weil es heißt: Einst besprachen sich die JHWH fürchten, jemand mit seinem Gefährten. (Mal 3,16) Woher [ist zu belegen], sogar bei einem? Weil es heißt: An jedem

7 Trennung zwischen Heiligem und Profanem am Ausgang des Schabbat. Bei der Havdala wird ein Segen über das Feuer über einem angezündeten Licht gesprochen.

8 Ein Gericht hat mindestens drei Richter und zwei Zeugen. 
Ort, an dem ich meines Namens gedenken lasse, werde ich zu dir kommen und dich segnen. (Ex 20,21)

Die Schechina bevorzugt es, sich bei Menschen aufzuhalten, die die Gebote gerne ausüben, wie in Jalkut zu Jona 1,3 hervorgehoben wird:

Rabbi Jona sagte: Jona, der Sohn Ammitais, war unter den Wallfahrern, und als er zur Freude[nfeier] von Beit-Haschoeba ${ }^{9}$ hinzutrat, ruhte der Geist der Heiligen auf ihm. Um dich zu lehren, dass die Schechina nur auf [dem] ruht, [der] freudigen Herzens [ist], denn es heißt: Und als der Saitenspieler spielte, kam die Hand JHWHs auf ihn. (II Reg 3,15)

Die Schechina, die zur Zeit des Tempels nur im Allerheiligsten wohnte, ruht damit nach Auffassung des Jalkut zum Zwölfprophetenbuch auf den Menschen, die die Gebote der Tora freudigen Herzens erfüllen und sich mit der Tora beschäftigen.

\subsubsection{Der Messias}

Auch die Messiasthematik tritt in Jalkut Schimoni zu den zwölf Propheten immer wieder in den Vordergrund. Anmerkungen zur Messiasthematik durchziehen die Auslegung von Hosea bis Maleachi, zuletzt findet sie sich in einer Auslegung zu Mal 3,23. Eine erste Anmerkung zum Thema bietet Jalkut Schimoni zu Hos 2,16, wo ein letzter Erlöser für Israel verheißen wird:

Deshalb, siehe, ich werde sie locken und sie in die Wüste führen. (Hos 2,16) R. Berekhja [sagte] im Namen von R. Levi: Wie der erste Erlöser ist der letzte Erlöser. Wie der erste Erlöser ihnen erschien und wieder vor ihnen verborgen wurde, ebenso der letzte Erlöser.

$\mathrm{Zu}$ Hos 2,17 heißt es im Jalkut Schimoni, 60.000 werden in den Tagen des Messias wieder ins Land Israel geführt werden:

Es heißt: Ich nehme euch einen aus einer Stadt mir zum Volk. (Ex 6,7) Und dort heißt es: Ich werde euch ins Land hineinführen, [...] und ich werde es euch geben. (Ex 6,8) Man schließt daraus [einen Analogieschluss von] eurem Hinausziehen zu eurem Hineinkommen ins Land: Wie dort 60.000, so auch hier 60.000. Und es steht geschrieben: Und sie antwortet dort wie in den Tagen ihrer Jugend. (Hos 2,17) Wie ihr Hinausziehen aus Ägypten zu 60.000, so ihr Hineinkommen in das Land zu 60.000, ebenso zu 60.000 in den Tagen des Messias.

Wer dieser Messias ist und wie die messianische Zeit zu erkennen ist, wird dann in Jalkut Am 9,11 beschrieben:

9 Marcus Jastrow, A Dictionary of the Targumim, the Talmud Babli and Yerushalmi, and the Midrashic Literature (London: Luzac, 1903), 1528: Quelle, an der am Sukkotfest das Wasser für das Gussopfer geschöpft wurde. Vgl. mSuk 5,1. 
Und an diesen Tagen werde ich die Hütte Davids aufrichten. (Am 9,11) R. Nachman sagte zu R. Isaak: Hast du gehört, wann Bar Nafle ${ }^{10}$ kommen wird? Er sagte zu ihm: Wer ist Bar Nafle? Er sprach zu ihm: Der Messias! Er sprach zu ihm: Du nennst ihn „Wolkensohn“ (בר נפילי)? Er sprach zu ihm: Ja, denn es steht geschrieben: Und an diesen Tagen werde ich die Hütte Davids aufrichten, die gefallen ist (הנופלת). (Am 9,11) Er sagte zu ihm: So sagte R. Jochanan: 1) In der Generation, in der der Sohn Davids kommt, werden die Schriftgelehrten vermindert werden, und der Rest [von ihnen]: Ihre Augen werden vor Verlangen und Schmerz vergehen, und Trauer und viele Bedrängnisse werden in der Welt sein, bevor das Erste vorüber ist, kommt bereits das zweite [Elend]. Unsere Lehrer lehrten: In der Jahrwoche ${ }^{11}$, in der der Sohn Davids kommt[, geschieht folgendes]: Im ersten Jahr wird dieser Vers in Erfüllung gehen: Und ich werde es auf eine Stadt regnen lassen und auf eine andere Stadt werde ich es nicht regnen lassen. (Am 4,7) Im zweiten werden Pfeile des Hungers ausgesendet werden. Im dritten wird der Hunger groß sein, und Männer, Frauen, Kinder und Fromme und Männer der Tat sterben, und die Tora ist wegen der sie Studierenden vergessen. Im vierten wird Sättigung und doch keine Sättigung sein. Im fünften wird eine große Sättigung sein, und die Tora wird wegen derer, die sie studieren, zurückkehren. Im sechsten sind [himmlische] Stimmen [zu hören]. Im siebten sind Kriege, am Ende des siebten kommt der Sohn Davids. R. Josef sagte: Wie viele Jahrwochen gab es, und er ist nicht gekommen! Abaje sagte zu ihm: Waren denn in der sechsten [Jahrwoche himmlische] Stimmen und in der siebten [Jahrwoche] Kriege? Und waren sie in der [richtigen] Reihenfolge da? R. Jehuda sagt: 2) In der Generation, in der der Sohn Davids kommt, wird das Versammlungshaus [der Gelehrten] zur Unzucht [benutzt] werden, und Galiläa wird zerstört und der Libanon verwüstet, und die Grenzbewohner werden von Stadt zu Stadt ziehen, und finden kein Erbarmen. Und die Weisheit der Schriftgelehrten verfliegt, und man weist Sündenfurcht von sich. Das Gesicht jener Generation ist wie das Gesicht eines Hundes ${ }^{12}$. Und die Wahrheit wird vollständig vertrieben (נעדרת) und wohnt in Herden (עדרים) in der Wüste, denn es heißt: Die Wahrheit wird vertrieben. (Jes 59,15) R. Nehorai sagt: 3) In der Generation, in der der Sohn Davids kommt, werden die Jungen die Alten beschämen, und die Alten werden gegen die Jungen aufstehen, eine Tochter tritt gegen ihre Mutter auf, eine Schwiegertochter gegen ihre Schwiegermutter und der Sohn schämt sich nicht vor seinem Vater. R. Nechemja sagte: 4) In der Generation, in der der Sohn Davids kommen wird, wird die Frechheit überhandnehmen und die Vornehmheit (היוקר) (ברר) vergeht. Der Weinstock wird keine Früchte bringen, und der Wein wird mit hohem Preis (ביוקר) [gehandelt], und die ganze Welt wendet sich der Häresie zu. Dies ist eine Stütze für R. Isaak, denn R. Isaak sagte: Der Sohn Davids kommt erst, wenn sich die ganze Welt der Häresie zugewandt haben wird (שמהפך), denn es heißt: Ist er komplett in weiß verwandelt (הפך), so ist er rein. (Lev 13,13) Die Rabbanan lehrten: Dass die Macht (י) verschwunden ist. (Dtn 32,36) [Der Sohn Davids kommt erst,] wenn die Denunzianten viele geworden sind. Eine andere Auslegung: [Der Sohn Davids kommt erst,] wenn die Weisen wenige geworden sind. Eine andere Auslegung: [Der Sohn Davids kommt erst,] wenn jede Peruta aus dem Geldbeutel verschwunden sein wird. Eine andere Auslegung: [Der Sohn Davids kommt

10 Samuel Krauss, Griechische und Lateinische Lehnwörter im Talmud, Targum und Midrasch, Bd. 2

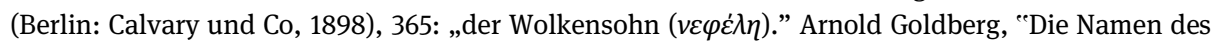
Messias in der rabbinischen Traditionsliteratur. Ein Beitrag zur Messianologie des rabbinischen Judentums." Mystik und Theologie des rabbinischen Judentums. Gesammelte Studien I, hg. Margarete Schlüter, Peter Schäfer (Tübingen: Mohr Siebeck, 1997), 208-274 hier 247-248.

11 Septennium: Periode von 7 Jahren, Vgl. Marcus Jastrow, A Dictionary of the Targumim, the Talmud Babli and Yerushalmi, and the Midrashic Literature (London: Luzac, 1903), 1510.

12 Das heißt: Frechheit und Unverschämtheit werden vorherrschen.

13 Marcus Jastrow, A Dictionary, 571 zu יוקר:: "nobility" und "high price." 
erst,] wenn sie an der Erlösung verzweifelt sein werden. Und Bewahrtes (עצור) und Bewährtes verschwunden ist. (Dtn 32,36) [Dann, wenn] Israel, wenn dies möglich wäre, keinen Helfer und Stützer hat. R. Zera sagte, wenn er die Rabbanan traf, zu ihnen: Ich bitte euch, sie ${ }^{14}$ nicht hinauszuschieben. Es wird gelehrt: Drei [Dinge] kommen, wenn man nicht daran denkt, und diese sind es: Der Messias, ein Fund, ein Skorpion. R. Levi sagte: Wenn du eine schmähliche und lästerliche Generation auf den Fuß des Messias spähen siehst, was ist der Grund dafür? [Gedenke, JHWH, der Schmach deiner Knechte ...] mit der deine Feinde, JHWH, dich verlästern, mit der sie die Fersen deines Messias verlästern. (Ps 89,51-52) Und danach steht geschrieben: Gepriesen sei JHWH ewiglich, Amen und Amen. (Ps 89,53)

Jeder Versuch, den Messias auf einem „einfachen Weg“ herbeizubringen, ist verboten und wird nicht erfolgreich sein. Der messianischen Zeit muss, wie hier gezeigt, großes Elend vorausgehen. Um die Bedeutungsschwere dieser Auslegung hervorzuheben, werden zwei Mal sieben ${ }^{15}$ Qualen beschrieben und in der zweiten und dritten Auslegung zusätzliche Meinungen über mögliche vorausgehende Qualen geäußert.

Die Ankündigung über das Kommen des Messias und die messianische Zeit setzt sich in Jalkut Micha fort. Zunächst werden zu Mi 1,15 und Mi 2,13 zwei mögliche messianische Figuren eingeführt. Diese werden nicht explizit benannt, sondern sind noch im Verborgenen. So heißt es zu Mi 1,15:

Ich werde dir noch den einen Erben bringen. (Mi 1,15) Sein Heiligtum und sein Königtum sind auf ewig. Bis Adulam kommen wird, (Mi 1,15) denn es heißt: Und Jehuda zog hinab von seinen Brüdern und schloss sich einem Mann, einem Adulamiter, an. (Gen 38,1)

Mi 1,15 wird stark beschnitten und auf seine für die Auslegung wichtige Kernaussage reduziert. Vollständig heißt es in Mi 1,15: Ich will dir noch, Maresa, den einen Erben bringen und die Herrlichkeit Israels soll bis nach Adulam kommen.

Die Auslegung an dieser Stelle impliziert das Wissen um die fehlenden Teile des Verses. So kann an dieser Stelle die „Herrlichkeit Israels“, die nach Adulam kommen wird, durchaus auch als messianische Figur verstanden werden und somit als der zuvor im gleichen Vers angekündigte eine Erbe.

Die Frage nach der Identität des Messias setzt sich in Jalkut Micha weiter fort. $\mathrm{Zu}$ Mi 2,13 wird gefragt, wer mit „Durchbrecher“ gemeint ist. Die Möglichkeit, neben Jakob auch den Messias in diesem Bild zu sehen, bleibt offen im Raum stehen:

Der Durchbrecher (הפורץ) steigt vor ihnen herauf. (Mi 2,13) In dieser Welt habe ich wegen Jakob die Mauer der Welt durchbrochen (פרצתי): Und der Mann breitet (ויפרץ) sich sehr aus. ${ }^{16}$ (Gen 30,43) R. Tanchuma bar Abba sagte: 120 Myriaden Schafe gehörten ihm, denn es heißt: Und der Mann breitet sich sehr aus. (Gen 30,43) Und es steht geschrieben: Und das Volk vermehrte sich und war sehr mächtig. (Ex 1,20) Wenn es dort 60 Myriaden sind, da [einmal] geschrieben steht:

14 Die messianische Zeit.

15 Die Zahl sieben als symbolisch bedeutend, vgl. z.B. sieben Tage der Schöpfung.

16 D.h. Er war sehr reich. 
Sehr, (Ex 1,20) sind es hier 120 Myriaden, denn es steht geschrieben: Sehr, sehr. (Gen 30,43) Und in der kommenden Zukunft durchbreche sie für deine Kinder, denn es heißt: Ein Durchbrecher (הפורץ) steigt auf. (Mi 2,13)

Nach dieser Einleitung, die bereits das Versprechen des Kommens des Messias und die anfängliche Thematisierung um seine Person beinhaltet, leitet Jalkut Mi 5,2 und Mi 5,4 die Beschreibungen der messianischen Leidenszeit ein. Dort heißt es:

Deshalb wird er sie hingeben bis zu der Zeit, in der sie geboren hat. (Mi 5,2) Rav Jehuda sagte im Namen Rabs: Der Sohn Davids wird erst kommen, wenn die frevelhafte Regierung sich neun Monate lang über die ganze Welt ausgedehnt hat, denn es heißt: Deshalb wird er sie hingeben bis zu der Zeit, in der sie geboren hat. (Mi 5,2) Und wir stellen ihm sieben Hirten und acht gesalbte Männer zur Seite. (Mi 5,4) Wer sind diese sieben Hirten? David in der Mitte, Adam, Seth und Methusalem zu seiner Rechten. Abraham, Jakob und Mose zu seiner Linken. Und wohin ging Isaak? Er ging und kam an die Pforte zur Gehenna, um seine Söhne aus dem Gericht der Gehenna zu retten. Wer sind die acht gesalbten Männer? Issai, Saul, Samuel, Amram ${ }^{17}$, Zephanja, Zedekia, Elija und der Messias.

In dieser Leidenszeit werden acht gesalbte Männer auftreten, bis es zur Erlösung kommt. Die Reihenfolge zeigt, dass Elija der letzte Prophet vor dem Kommen des Messias ist. Auch die Gesamtauslegung zum Jalkut zu den zwölf Propheten endet mit Elija als dem Vorboten des Messias.

Der Jalkut zum zweiten Kapitel von Habakuk hat warnenden Charakter. Nachdem bereits mehrfach die Zusage des am Ende stehenden Heils gegeben wurde, wird der Leser an dieser Stelle eindringlich gewarnt, auch in der Zeit der Not und des Unglücks nicht mit Gott und dessen Vorhaben zu hadern. ${ }^{18}$ In der Auslegung zu Hab 2,3 wird die Mahnung expliziter. Hier wird deutlich verboten, sich jeglicher Endzeitberechnung hinzugeben:

Was [bedeutet]: Und es eilt zum Ende [und] lügt nicht? (Hab 2,3) R. Samuel bar Nachmani sagte: Es schwinde der Geist derer, die das Ende berechnen, denn sie sagen: Wenn das Ende erreicht sein wird und es [dann] nicht gekommen ist, dann kommt es nicht mehr. Vielmehr harre man seiner, denn es heißt: Wenn es sich verzögert, harre seiner. (Hab 2,3) Vielleicht sagst du: Wir harren, aber er harrt nicht. Die Bibel lehrt: Und deshalb harrt JHWH, um sich eurer zu erbarmen, und deshalb wird er sich still verhalten, um sich eurer zu erbarmen, denn ein Gott des Gerichts ist JHWH. (Jes 30,18) Wenn es sich verzögert, [und es so ist,] dass er harrt und wir harren, wer hält es dann zurück? Die göttliche Eigenschaft des Gerichts hält es zurück. Wenn es sich verzögert, [und es so ist,] dass die göttliche Eigenschaft des Gerichts es zurückhält, warum harren wir? Um eine Belohnung zu erhalten, denn es heißt: Glücklich sind alle, die seiner harren. (Jes 30,18)

17 In der Quelle heißt es „Amos“.

18 Vgl. dazu auch die Auslegung zu Hab 1,2-3 und Hab 2,2. Hab 1,2-3 wird „thematisch passend“ ins zweite Kapitel verlegt, um die Auslegungen Hab 2,2 und Hab 2,3 passend anschließen zu können. 
Die „Endzeit“, die Zeit des Heils, bricht an, wenn Gott dies will. Durch den gesamten Jalkut zu den zwölf Propheten wird deutlich, dass das Kommen des Messias durch bestimmte vorausgehende Ereignisse angezeigt wird, sich aber nicht zeitlich bestimmen lässt. Es geht, wie Jalkut Zeph 3,8 formuliert, darum, auf Gottes Einschreiten zu warten:

\begin{abstract}
Deshalb wartet auf mich, Spruch JHWHs, bis zum Tag, an dem ich mich als Zeuge erhebe. $($ Zeph 3,8) Der Heilige, gepriesen sei er, sagte: Ein Schwur ist vor mir: Für jeden, der auf mein Königreich wartet, werde ich selbst zum Guten zeugen, denn es heißt: Deshalb wartet auf mich, Spruch JHWHs, bis zum Tag, an dem ich mich als Zeuge erhebe. (Zeph 3,8) Diese sind die Trauernden Zions, die ihren Geist erniedrigen lassen und ihre Beschämung hören und schweigen und es sich nicht als Verdienst für sich selbst anrechnen. Eine andere Auslegung: Bis zum Tag, an dem ich mich als Zeuge erhebe. (Zeph 3,8) An dem Tag, an dem ich mich erhebe, um den Messias zu bezeugen, dessen Verdienst meinen ganzen Hausstand (familia) aufwiegt. Sind doch all diese Maßgaben [vor mir] und ihr konntet nicht auf mich warten! Der Heilige, gepriesen sei er, sagte zu ihnen: Ihr Lohn ist verdoppelt: Denn das Volk, das in Zion und in Jerusalem wohnt ... Der Gnädige [wird dir gnädig sein]. (Jes 30,19) Denn er gab euch den Lohn der Tora. Er wird dir gnädig sein. (Jes 30,19) Dass sie die Tage des Messias genießen. Wenn er es hört, erhört er dich. (Jes 30,19) In Bezug auf die kommende Welt. Alles ist durch das Verdienst des Messias, der all jene Jahre zurückgehalten wurde.
\end{abstract}

Klar ist von hier, dass das Kommen des Messias durch Sünden zurückgehalten wird. Daher rückt die Umkehr von Sünden, ab Jalkut Sacharja, in den Fokus. Das gesamte erste Kapitel in Sacharja beschränkt sich allein auf das Thema der Umkehr. Zusätzlich greift der Jalkut zu Sacharja $2-4^{19}$ die Frage nach der Identität des Messias auf. So heißt es zu Sach 2,3:

Und JHWH zeigte mir vier Schmiede. (Sach 2,3) Wer sind diese vier Schmiede? R. Chama bar Bisna sagte, R. Simeon Chasida sagte: Der Messias, Sohn Davids, der Messias, Sohn Josefs, Elija und der rechtschaffene Priester.

Um die zwei Messiasfiguren geht es auch in Jalkut Sach 4,3 und Sach 4,7, wenn ,zwei Ölbäume“ und „großer Berg“ als Metaphern für Messiasfiguren gedeutet werden:

Und zwei Ölbäume ihm gegenüber. (Sach 4,3) Dies sind die zwei Gesalbten: Der Kriegsgesalbte und der zum König über Israel Gesalbte.

Wer du auch bist, großer Berg, vor Serubbabel [wirst du] zur Ebene. (Sach 4,7) Das ist der König, der der Messias ist. Warum bezeichnet man ihn als „großen Berg“? Weil er größer ist als die Väter, denn es heißt: Siehe, mein Knecht wird glücklich sein, erhaben wird er sein und erhöht und sehr hochgestellt. (Jes 52,13) Erhabener als Abraham, denn es heißt: Erhoben habe ich meine Hand zu JHWH. (Gen 14,22) Und erhöhter als Mose, denn heißt es über ihn: Trage es in deinem Schoß. (Num 11,12) Und hochgestellter als die Dienstengel, denn über sie steht geschrieben: Und ihre Länge und ihre Höhe war zum Erschrecken. $(E z 1,18)$ Und aus wem ging er hervor? Aus David,

19 Sach 2,$3 ; 4,3 ; 4,7$. 
denn es heißt: Und der Sohn Salomons war Rehabeam, sein Sohn war Abija, sein Sohn war Asa, sein Sohn war Jehoschafat. (I Chr 3,10) Bis: Und die Söhne von Elioenai waren Hodawjahu und Eljaschib und Pelaja und Akub und Jochanan und Delaja und Anani, sieben. (I Chr 3,24) Bis hier benennt die Schrift ausdrücklich [alle Namen] bis Anani (ענני). Wer ist Anani? Das ist der Messias, denn es heißt: Ich schaute im Gesicht der Nacht und siehe, mit den Wolken (ענני) des Himmels [war er gekommen]. (Dan 7,13) Und was ist [die Bedeutung von] „sieben“? Was in Bezug auf den Messias geschrieben ist, wenn es heißt: Denn wer den Tag der kleinen [Taten] geringschätzt, wird sich doch freuen, wenn er den Trennstein sieht: In Serubbabels Hand sind sieben. (Sach 4,10 ) Und deshalb heißt es: Wer du auch bist, großer Berg, vor Serubbabel [wirst du] zur Ebene. (Sach 4,7) Das ist der, über den geschrieben steht: Und er richtet mit Gerechtigkeit die Armen und entscheidet mit Geradheit. (Jes 11,4)

Bereits in den Schriften der tanaanitischen Zeit und im Targum sind zwei Messiasfiguren zu belegen. ${ }^{20}$ Ein Messias ben Josef muss im endzeitlichen Kampf sterben, bevor der Messias ben David die Erlösung bringt. In Jalkut Sach 9,1 heißt es, dass der Messias die Völker und Israel richten wird:

Ein Spruch: Das Wort JHWHs über das Land Hadrach (חדרך) und Damaskus, seine Wohnstätte. (Sach 9,1) R. Jehuda erklärte: Das ist der Messias, denn er ist scharf (חד) zu den Völkern (אומות) der Welt und mitfühlend (רך) zu Israel.

Obwohl der Messias ein König ist, wird er sich mit den Armen und Kranken solidarisieren, wie der Jalkut zu Sach 9,9, hervorhebt:

Arm und auf einem Esel reitend. (Sach 9,9) Das ist der Esel, das Fohlen der Eselin, die in der Dämmerung ${ }^{21}$ geschaffen wurde. Das ist der Esel, den unser Vater Abraham zur Bindung seines Sohnes Isaaks sattelte. Das ist der Esel, auf dem unser Lehrer Mose ritt, als er nach Ägypten kam, denn es heißt: Und er ließ sie auf einem Esel reiten. (Ex 4,20) Das ist der Esel, auf dem in der Zukunft der Sohn Davids reiten wird. R. Jehoschua ben Levi traf Elija, der am Eingang der Höhle von R. Simeon ben Jochai saß. Er sagte zu ihm: Wann wird der Messias kommen? Er sagte zu ihm: Geh und frage ihn selbst! Er sagte zu ihm: Und wo wohnt er? Er antwortete ihm: Am Eingang von Rom. Er sagte zu ihm: Und was sind seine Zeichen? Er antwortete ihm: Er wohnt unter den Armen und mit Krankheit Beladenen. Sie lösen alle [ihre Bandagen] und verbinden sie [neu] mit einem Verband. Er aber er löst [nur jeweils] eine [Bandage] und verbindet sie [neu]. Er sagt: Vielleicht werde ich gebraucht, und ich darf mich nicht verspäten. Er ging zu ihm und sagte zu ihm: Friede sei dir, mein Lehrer und mein Herr! $\mathrm{Er}^{22}$ antwortete: Friede sei mit dir, Sohn von Levi. Er sagte zu ihm: Wann kommt mein Herr? Er sagte zu ihm: Heute! Am nächsten Tag traf er Elija. Er ging zu ihm und sagte zu ihm: Was hat er dir gesagt? Er sagte zu ihm: Er hat mich mit Lügen

20 Gerald J. Blidstein, „Messiah“, in Encyclopaedia Judaica, hg. Michael Berenbaum, Fred Skolnik, 2. Aufl., Bd. 14 (Detroit: Macmillan Reference USA, 2007), „Messiah in Rabbinic Thought“, 110-115; Louis Goldberg, „Der messianische Gedanke im Judentum“, in Tod eines Messias. Messiasgestalten und Messiaserwartungen im Judentum, hg. Kai Kjær-Hansen, (Neuhausen: Hänssler, 1996), 92-101; Gershom Scholem, „Zum Verständnis der messianischen Idee im Judentum“, in Judaica I, hg. Gershom Scholem, (Frankfurt am Main: Suhrkamp, 1963), 7-74.

21 Bevor nach den Schöpfungstagen der Schabbat anbrach.

22 Der Messias. 
belogen. $\mathrm{Er}^{23}$ sagte $\mathrm{zu}$ ihm: Was hat er dir gesagt? Er sagte zu ihm: Ich sagte ihm: Friede sei mit dir, mein Lehrer und mein Herr. Und er sagte: Friede sei mit dir, Sohn von Levi. [Elija] sagte zu ihm: Ich sehe, dass er dir versichert hat, dass du und dein Vater unter den Kindern der zukünftigen Welt seid. Daraufhin sagte ich zu ihm: Wann kommt der Messias? Er sagte zu mir: Heute! [Elija] sagte zu ihm: Heute, wenn ihr auf seine Stimme hört. (Ps 95,7) Alexandri sagte, R. Jehoschua ben Levi verwies auf [den Widerspruch]: Es steht geschrieben: Und siehe, mit den Wolken des Himmels [kam einer wie der Sohn eines Menschen]. (Dan 7,13) Und es steht geschrieben: Arm und auf einem Esel reitend. (Sach 9,9) Haben sie Verdienst: Mit den Wolken des Himmels. (Dan 7,13) Haben sie kein Verdienst: Arm und auf einem Esel reitend. (Sach 9,9) König Schapur sagte zu Samuel: Du sagtest, dass der Messias auf einem Esel kommt. Ich werde ihm ein weißes Pferd schicken, das mir gehört. Er sagte zu ihm: Welches von weißer Farbe gehört dir? ${ }^{24}$

Dass Elija als direkter Vorbote des Messias fungieren wird, ist noch einmal Thema des Jalkut zu Mal 3,23. Aber auch der Lockruf eines seltenen Vogels, wird das Kommen des Messias ankündigen, wie der Jalkut zu Sach 10,8 ausführt:

Ich locke (אשרקה) 25 sie herbei und versammle sie. (Sach 10,8) ... Abaje sagte: Das ist doch [der Vogel], von dem man meint, dass er, wenn er sitzt [das Geräusch] „schrikrik“ (שריקריק) macht. Abaje sagte: Es ist eine überlieferte Tradition, dass, wenn er auf dem Boden sitzt und „schrikrik“ macht, der Messias sofort kommt, denn es steht geschrieben: Ich locke (אשרקה) sie herbei und versammle sie, denn ich will sie erlösen. (Sach 10,8)

Die Messiasthematik beendet der Jalkut mit einem Vers, in dem Gott selbst eine Zusage an ganz Israel ausspricht: Gott lockt Israel zu sich, er will es versammeln, denn er will Israel erlösen. Damit treten sowohl die Propheten, als auch die Messiasfiguren als Boten in den Hintergrund, denn Gott selbst wird die Erlösung herbeiführen.

Die hier behandelten Themen zeigen, dass der Autor des Jalkut zusätzlich zu der fortlaufenden Auslegung predigtartig Themen von Hosea bis Maleachi entwickelt, die nicht als zusammenhängend erkannt werden, wenn die Auslegung des Jalkut zu den einzelnen zwölf Schriften der kleinen Propheten als unabhängige Einzelschriften gelesen werden.

23 Elija.

24 Siehe Hyman/Schiloni, JS Sacharja, 810 Anm. 16, die darauf verweisen, dass Raschi zu bAZ 30a die Stelle anders interpretiert. Er deutet rיור als persisches Wort für die Zahl 100: „ein hundertfarbiges Pferd“. Vgl. The Babylonian Talmud. Seder Nezikin. Sanhedrin, translated into English by H. Freedman (London: Soncino Press, 1935), 664: "Have you a hundred-hued steed?"

25 Hapax legomenon. 